\title{
Assessment of microscope-integrated indocyanine green angiography during intracranial aneurysm surgery: A retrospective study of $\mathbf{I} 20$ patients
}

\author{
Jin Li, ZhiGang Lan, Min He, Chao You \\ Department of Neurosurgery, West China Hospital, Sichuan University, 37 Guo Xue Xiang Street, Chengdu - 610 041, China
}

\author{
Address for correspondence: \\ Dr. Min He, \\ Department of Neurosurgery, \\ West China Hospital, \\ Sichuan University, \\ 37 Guo Xue Xiang Street, \\ Chengdu, Sichuan - 610041 , China. \\ E-mail: hxswjim@163.com
}

DOI: $10.4103 / 0028-3886.55607$

\begin{abstract}
Aims: The purpose of this study was to assess the clinical value of indocyanine green angiography (ICG) in intracranial aneurysm surgery by comparing the findings with postoperative angiographic results. Materials and Methods: One hundred and twenty patients with 148 intracranial aneurysms were included. ICG angiography was performed before and/or after the aneurysm clipping. A near-infrared excitation light illuminated the operation field, ICG was injected intravenously. The intravenous fluorescence was imaged with a video camera integrated into the microscope. Results: A total of 208 investigations of ICG angiography were performed. Aneurysm clipping was applied in 120 patients. Incomplete clipping was detected in four patients. Parent and/or branching artery stenosis was found in five patients. Delayed perfusion of ICG was detected in one patient. Postoperative digital subtraction angiography (DSA) was performed in 108 patients. The postoperative angiographic results were consistent with findings on intraoperative ICG angiograms in 100 patients $(92.6 \%)$. In three cases, a mild stenosis was seen on DSA, which was not detected intraoperatively using ICG angiogram. In one patient, middle cerebral artery stenosis was found. Three patients had small residual aneurysms found by postoperative DSA. The remaining one developed a severe cerebral vasospasm. Conclusions: ICG angiography is a simple, reliable and cost-effective method. It provides real-time information in detecting the patency of parent, branching, perforating arteries and residual aneurysm. This technique may be a useful adjunct to improve the Quality of intracranial aneurysm surgery.
\end{abstract}

Key words: Cerebral angiography, indocyanine green angiography, intracranial aneurysm

\section{Introduction}

The purpose in the surgical clipping of intracranial aneurysm is to completely occlude the lumen of the aneurysm and maintain the patency of the parent, branching and perforating arteries. However, vascular occlusion of either parent, branching or perforating arteries due to improper clip placement during intracranial aneurysm surgery is still fairly frequent. Postoperative angiograms in several studies have revealed an incidence of vascular occlusion between 4 and $12 \%$, residual aneurysm filling between 2 and $8 \% .^{[1,2]}$ Indocyanine green angiography (ICG) has been used in recent years. ${ }^{[1-3]}$ Raabe et al. reported their initial experience with ICG angiography in intracranial aneurysm surgery. ${ }^{[4-6]}$ We present the results of our study to assess the clinical value of intraoperative ICG angiography in verifying the vascular patency and residual aneurysms, and to identify factors that could predispose patients to postoperative complications. 


\section{Materials and Methods}

This study was performed in the Department of Neurosurgery, West China Hospital. The institutional review board of West China Hospital approved this study. Written informed consent was obtained. The ICG angiography studies were compared with the postoperative digital subtraction angiography (DSA) results.

\section{Patients and clinical characteristics}

Between January 2008 and December 2008, 386 patients with aneurysmal subarachnoid hemorrhage (SAH) were admitted to West China Hospital and were enrolled in our Subarachnoid Hemorrhage Outcomes Project. Of these patients, 120 cases harboring 148 aneurysms which were treated with surgical clipping using ICG angiography intraoperatively were included. The demographic and clinical characteristics of 120 patients are listed in Table 1 . There were 48 males and 72 females with a mean age of 54.1 years (range 29-81 years). Eighty-four patients (70\%) presented with spontaneous SAH, 36 patients $(30 \%)$ were without SAH. Forty-eight aneurysms involved the anterior cerebral artery (ACA) or anterior communicating artery (AComA) region. Sixty-two aneurysms involved the posterior communicating artery (PComA) and internal carotid artery (ICA). Twenty-two aneurysms were located in middle cerebral artery (MCA). There were 16 aneurysms in the posterior circulation. The average size of 148 aneurysms was $8.2 \mathrm{~mm}(1.0-25.8 \mathrm{~mm})$ based on angiography measurement. Multiple aneurysms were found in 20 patients.

\section{Procedures}

In addition to our standard protocol for intracranial aneurysm surgery, a history of iodine allergy, pregnancy, previous anaphylactic reactions to contrast media or dye injection was acquired either from the patients or their relatives depending on the clinical condition and written informed consent was obtained. The surgical procedure was performed as usual. Intraoperatively, the choice of ICG angiography was mainly based on the surgeon's decision. In our practice, if the aneurysm was giant and complex, ICG angiography was used before and after clipping. For small and simple aneurysms, ICG angiography was not routinely performed after clipping. If abnormal finding was noted, the procedure was repeated after $15 \mathrm{~min}$. The routine dose for ICG cerebral angiography was $0.2-0.5 \mathrm{mg} /$ $\mathrm{kg}$ up to a maximum dose of $5 \mathrm{mg} / \mathrm{kg}$.

\section{Principles and technique of indocyanine green angiography video angiography}

The ICG-integrated microscope (Leica M525 OH4, Germany) was introduced to our institution in 2008. ICG

\begin{tabular}{lc}
\hline $\begin{array}{l}\text { Table 1: Demographic and clinical characteristics in } 125 \text { patients } \\
\text { with } 148 \text { aneurysms } \\
{ }^{\circ}\end{array}$ \\
\hline \multicolumn{2}{l}{ Feature value (\%) } \\
\hline No. of patients & 120 \\
No. of aneurysms & 148 \\
Sex (F/M) & $48 / 72$ \\
Mean year (range) & 53 yr (35-79 yr) \\
SAH & $36(30)$ \\
No SAH & $84(70)$ \\
SAH (H and H) & $20(24)$ \\
I & $15(17)$ \\
II & $25(30)$ \\
III & $16(19)$ \\
IV & $8(10)$ \\
V & \\
Location of aneurysms & $48(32)$ \\
ACA/AComA & $62(42)$ \\
ICA/PComA & $22(15)$ \\
MCA & $16(11)$ \\
Post circulation & $8.2 \mathrm{~mm}(1.0-25.8 \mathrm{~mm})$ \\
Mean aneurysm size (range) & $20(17)$ \\
Multiple aneurysms
\end{tabular}

$\mathrm{H}$ and $\mathrm{H}$-Hunt and Hess classification; ACA-Anterior cerebral artery; AComA-anterior communicating artery; ICA-Internal carotid artery; PComA-Posterior communicating artery; MCA-Middle cerebral artery; SAH-Subarachnoid hemorrhage

is a near-infrared fluorescent tricarbocyanine dye, which is widely used in ophthalmology for assessment of retinal microcirculation. The absorption and emission peaks of ICG dye (805 and $835 \mathrm{~nm}$, respectively) lie within the optical window of tissue in which absorption attributable to endogenous chromophores is low. After the ICG has been injected intravenously, ICG is bound to globulins ( $\alpha 1$ lipoproteins) within one to two sec. ICG is not metabolized in the body, and it is eliminated almost completely in the liver. The plasma half-time is three minutes. The routine recommended dose for cerebral angiography is $0.2-0.5 \mathrm{mg} / \mathrm{kg}$, the maximal daily dose should not exceed $5 \mathrm{mg} / \mathrm{kg}$. The operative field is illuminated by a light source that has a wavelength covering part of the ICG absorption band. The ICG dye (Dandong Pharmacy Company, China) is injected as a bolus (standard dose of $25 \mathrm{mg}$ dissolved in $5 \mathrm{ml}$ water). The ICG-induced fluorescence signal is captured and recorded by the video camera integrated into the microscope. About 7-8 sec following intravenous ICG injection, the arteries begin to fill, followed by the small arteries, cortical veins. The time course of ICG angiography could be divided into arterial, capillary and venous phase as observed in DSA angiography. Approximately 1-2 min later, the intensity of ICG gradually decreased. The time allows the surgeon to assess and adjust the clip. The image is stored in the video camera and can be reviewed repeatedly.

\section{Postoperative digital subtraction angiography}

In 120 patients treated with surgical clipping, eight patients died as a consequence of SAH. DSA examination was not performed on four patients due to their poor medical conditions. Thus, postoperative DSA was performed in 108 patients between Day one and Day 
seven. Standard projection images were obtained and reviewed. The DSA results were compared with intraoperative ICG angiogram findings.

\section{Results}

A total of 208 investigations of ICG angiography were performed. Aneurysm clipping was applied in 120 patients. ICG angiogram was performed before clip application in 58 cases, after clip application in 125 cases, and after clip adjustment in 25 cases. The maximum number of ICG applications for a patient was five. There were no ICG-related complications. The image quality and spatial resolution were excellent in 202 applications, allowing real-time assessment of the vascular patency. Failure of image was due to the diffuse bleeding from the surgical field in five applications, and in three cases was attributed to limited exposure of branching vessels.

Following intraoperative ICG angiography, abnormal findings were seen in ten patients [Table 2] and the outcomes are summarized in Table 3. Four of the ten patients had residual aneurysms and these included one ICA artery, two AComA and one PComA aneurysm. After adjusting the clips, the residual aneurysm was completely occluded. Repeated ICG angiography confirmed complete obliteration of the aneurysm. Parent and/or branching artery stenosis was found in five patients, of which two had MCA, one each had anterior choroidal artery (AchA) and AcomA aneurysms. The fifth patient (Case 4) had a PComA aneurysm and ICG angiography after initial clip application showed kinking and occlusion of the AChA. The placement of the clip was corrected and the arterial patency was restored. Delayed perfusion of ICG was detected in one patient (Case 2) with a large ophthalmic artery aneurysm originating from the atherosclerotic ICA with a plaque. The clip was not repositioned assuming that the plaque was affecting the image quality, however, the patient developed a postoperative MC infarction with poor outcome.

The postoperative angiographic results of 108 patients were consistent with findings on intraoperative ICG angiograms in 100 patients (92.6\%). In three cases, a mild stenosis was seen on DSA, which was not detected intraoperatively using ICG angiogram. However, these patients were asymptomatic. In Case 2, the delayed filling of ICG resulted in MCA stenosis confirmed by postoperative DSA and the patient suffered MCA infarction. Incomplete clipping was found in three patients by postoperative DSA, these residual aneurysms were missed by ICG angiography intraoperatively. For their small size, these aneurysms were not retreated and the patients were under follow-up observation. One patient

\begin{tabular}{|c|c|c|c|c|}
\hline \multirow[t]{2}{*}{ Age (y)/Sex } & \multicolumn{2}{|c|}{ SAH grade } & \multirow{2}{*}{$\begin{array}{l}\text { Aneurysm } \\
\text { location }\end{array}$} & \multirow[t]{2}{*}{ Size $(\mathrm{mm})$} \\
\hline & Fisher & Hunt-Hess & & \\
\hline $45 / F$ & 0 & 0 & Rt-MCA & $9.3 \times 16.0$ \\
\hline $52 / \mathrm{F}$ & 1 & 2 & Rt-OPhA & $12.0 \times 15.0$ \\
\hline $44 / M$ & 0 & 0 & Lt-ICA & $25.0 \times 21.0$ \\
\hline $60 / \mathrm{F}$ & 1 & 3 & Lt-PComA & $10.0 \times 8.0$ \\
\hline $70 / F$ & 2 & 2 & Lt-PComA & $12.0 \times 5.0$ \\
\hline $64 / M$ & 2 & 2 & AComA & $4.7 \times 2.8$ \\
\hline $50 / \mathrm{F}$ & 3 & 1 & AComA & $5.4 \times 2.8$ \\
\hline $56 / F$ & 3 & 1 & $\mathrm{AChA}$ & $4.0 \times 3.0$ \\
\hline $62 / F$ & 4 & 2 & AComA & $4.5 \times 5.5$ \\
\hline $33 / F$ & 0 & 0 & Rt-MCA & $6.0 \times 2.1$ \\
\hline
\end{tabular}

MCA-Middle cerebral artery; OPhA-Ophthalmic artery; ICA-Internal carotid artery; PComA-Posterior communicating artery; AComA-Anterior communicating artery; AChA-anterior choroidal artery

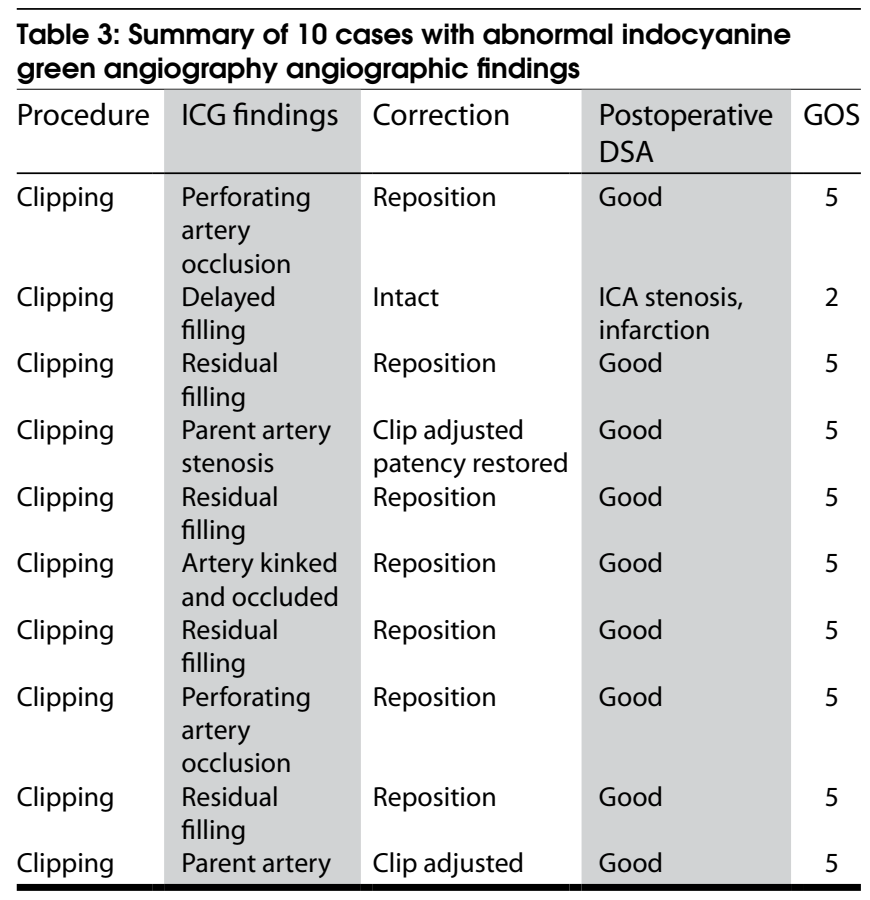

(Case 5) with a PComA aneurysm suffered severe cerebral vasospasm confirmed by postoperative DSA and aggressive hyperdynamic therapy was used without improvement.

\section{Discussion}

The most serious complications of intracranial aneurysm surgery are related to improper clip placement. Improper clip placement has been associated with compromised blood flow, and in a large number of cases, with subsequent development of delayed neurological deficit, stroke, and even death ${ }^{[3]}$ MacDonald et al. reported that $11.5 \%$ of their cases had compromised blood flow due to accidental vessel stenosis and $10.3 \%$ eventually suffered a serious stroke or death. ${ }^{[6]}$ Alexander et al. found that approximately $12 \%$ of cases with intracranial aneurysm 
clipping were associated with unexpected residual aneurysm, and unexpected major arterial occlusion. ${ }^{[1]}$ Despite great attempts to preserve the patency of the important vessels, intraoperative visual inspection is still not sufficient to reveal arterial compromise or occlusion with subsequent high occurrence rate of postoperative complications.

Intraoperative vascular imaging is very valuable and could be effective in decreasing the morbidity of intracranial aneurysm surgery. Various detecting methods, such as intraoperative microvascular Doppler ultrasonography and intraoperative DSA could provide valuable information about the parent or branching vessels' occlusion, residual aneurysm. ${ }^{[7]}$ Intraoperative angiography is the gold standard for the assessment of complete aneurysm clipping and vessel patency. Several studies have showed that intraoperative DSA reveals a significant number of cases of unexpected stenosis or occlusion, residual aneurysm filling. ${ }^{[8-10]}$ However, it is expensive, time-consuming, technically demanding, requires major logistical support and is still associated with a complication rate of $0.4-2.6 \% .^{[9]}$ The most important drawback of DSA is the ability to assess the patency of small perforating arteries.

ICG angiography has been widely used in ophthalmology for assessment of retinal microcirculation for more than 30 years, the complication rate is less than $0.1 \% \cdot{ }^{[11,12]}$ It is being used in recent years for cerebrovascular angiogram. This method is simple and quick, offering real-time information on the patency of arterial and venous vessels of all relevant diameters, including vessels of diameter less than $1 \mathrm{~mm}$ that are visible and not covered by clots or brain tissue. The ICG angiography integrated into the surgical microscope has considerably improved the simplicity and speed of the procedure. The procedure can be repeated after $15 \mathrm{~min}$. There is no need to remove the microscope from the surgical field or interrupt the operative manipulation. The available time of ICG angiography is two min, the timeframe allows the surgeon to act immediately to adjust the clip before the occlusion occurs. ICG angiography is most helpful in dealing with small vessels which are kinked or bent. ${ }^{[7-10]}$

The main limitation of ICG angiography is that the views are restricted to the field of the microscope. Vessels covered by blood clots, brain tissue or clip cannot be observed. When residual aneurysm neck is covered by parent artery or clips, the possibilities of residual aneurysm cannot be excluded. So, it is difficult to identify these situations intraoperatively. However, incomplete occlusion can be clearly diagnosed by the slow contrast filling. The assessment requires more angiographic views to identify the situation. ${ }^{[5]}$ Slight retraction of brain tissue or arteries may help the exposure of aneurysm neck or small arteries. The image of ICG angiography may be affected by calcifications and thick-walled atherosclerotic vessels or by partially or completely thrombosed aneurysms. The signal may be poor and the possibility of vascular stenosis cannot be excluded. For such cases, intraoperative DSA remains the modality of choice.

Several factors predispose a patient to improper clip placement affecting the ICG angiographic results and in our study, the anatomic location was one such factor. Aneurysms of posterior communicating artery, internal carotid artery, middle cerebral artery bifurcation, anterior choroidal artery were most likely occluded. In Case 3, the preoperative DSA showed a large ICA aneurysm with wide neck and downward projection [Figure 1a]. The aneurysm was exposed and a coincidental small AChA aneurysm was identified [Figure 1b]. ICG angiography was done before clipping [Figure 1c]. Complete clipping of the two aneurysms was performed [Figure 1d]. However, visual inspection could not verify the patency of the parent artery. ICG angiography eventually confirmed the complete clipping and the good filling of the parent artery after several clip repositioning [Figure 1e]. But the small anterior choroidal artery occlusion was overlooked as it was covered by the clips in ICG imaging, which resulted in a small infarction of the caudate nucleus confirmed by CT [Figure 1f]. The complex anatomy of these arteries with the presence of small-caliber and multiple adjacent perforators might affect the imaging results leading to improper clip placement.

Aneurysm size and projection were other factors in our study predisposing to vascular occlusion. We found that large and giant aneurysms were more frequently clipped improperly due to poor ICG angiographic imaging. In Case 2, the aneurysm originated from the ICA and projected downward. The anterior clinoid process and optic nerve partially covered the proximal neck. The exposure of the aneurysm was difficult. Two fenestrated clips in tandem were applied and visual inspection verified the complete clipping and the patency of the ICA. ICG angiography demonstrated the sluggish filling and weak intensity of the ICG. Considering the atherosclerotic plaque and thick walls of the artery, we left the clips in place and the patient suffered MCA infarction postoperatively. In the case of large aneurysm with downward projection, complete clipping of the neck and reconstruction of the arterial lumen were not easy and postoperative occlusion occurred more frequently. ${ }^{[7]}$ In such cases, intraoperative DSA is a more useful technique for successful clipping. ${ }^{[11,12]}$ 

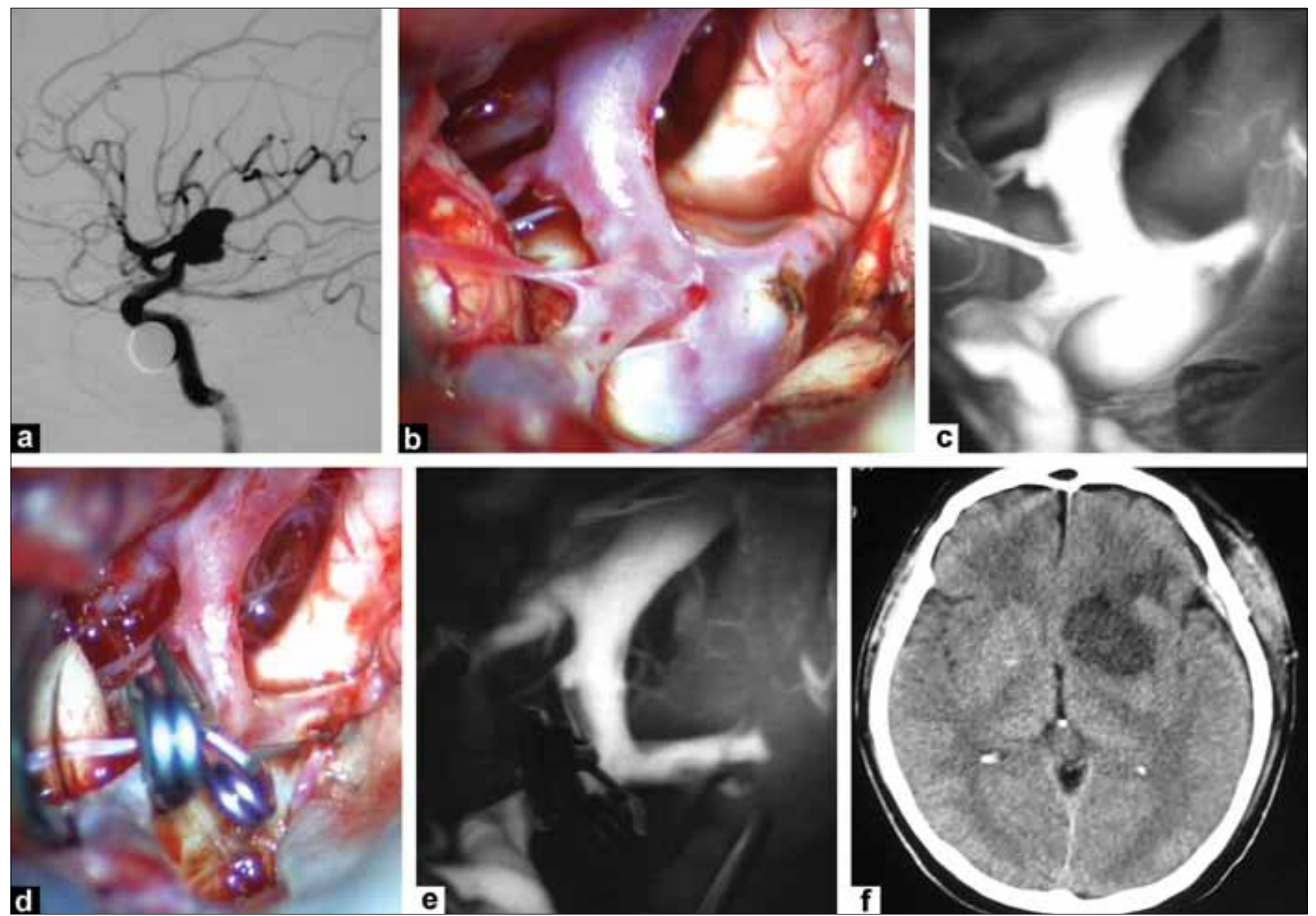

Figure 1: (a) Preoperative digital subtraction angiography image showing a left-side large internal carotid artery bifurcation aneurysm; (b) Intraoperative exposure of the internal carotid artery aneurysm and a coincidental small AChA aneurysm; (c) Indocyanine green angiography before clipping showing the aneurysms and all the relevant arteries; (d) Anatomic view after clipping; (e) Indocyanine green angiography after clipping showing the patency of

the parent artery and the anterior choroid artery is covered by clip; (f) Postoperative CT showing the infarction of the caudate nucleus

The parent artery with calcification, atherosclerotic plaque and thick wall may affect the ICG angiographic results. A delayed perfusion and sluggish arterial filling may be observed. In Case 2, the atherosclerotic plaque on the ICA was obvious and the imaging was observed about $15 \mathrm{sec}$ after ICG injection. The ICG fluorescence signal was poor. Considering that the atherosclerotic plaque might affect the imaging quality, we did not reposition the clips. The patient suffered infarction postoperatively. Our experience showed that any visible delay in perfusion seen on ICG angiography after clipping should raise the suspicion of stenosis or occlusion of the arteries. Intraoperative DSA should be performed to further verify the patency of the arteries in such patients.

A false-negative outcome was found in one patient with a PComA aneurysm. The preoperative DSA revealed a small PComA aneurysm [Figure 2a]. The operation was uneventful, visual inspection found the aneurysm was completely clipped, the patency of ICA and AChA was well maintained
[Figure 2b]. The ICG angiography also verified the results [Figure 2c]. However, the patient developed postoperative cerebral infarction confirmed by CT scan [Figure 2d]. Postoperative DSA demonstrated severe cerebral vasospasm [Figure 2e]. We thought the stenosis might be hemodynamically relevant. The etiology of this finding was unclear.

In our cohort, the postoperative angiographic results of 108 patients were consistent with findings on intraoperative ICG angiograms in 100 patients (92.6\%). Four $(3.7 \%)$ cases developed mild vascular stenosis or perforator occlusion. Three $(3 \%)$ had small residual aneurysms which were missed by ICG angiography intraoperatively. One $(1 \%)$ was hemodynamically relevant severe cerebral vasospasm. Payner et al. reported a $7.9 \%$ rate of residual aneurysms and a $4.8 \%$ rate of distal arterial branch occlusion. ${ }^{[13]}$ Dashti et al. studied a series of 190 patients with 239 intracranial aneurysms and revealed a $6 \%$ rate of unexpected neck residuals and a $6 \%$ rate of major and minor branching arteries ${ }^{[14]}$ In our study, the results of ICG angiography 

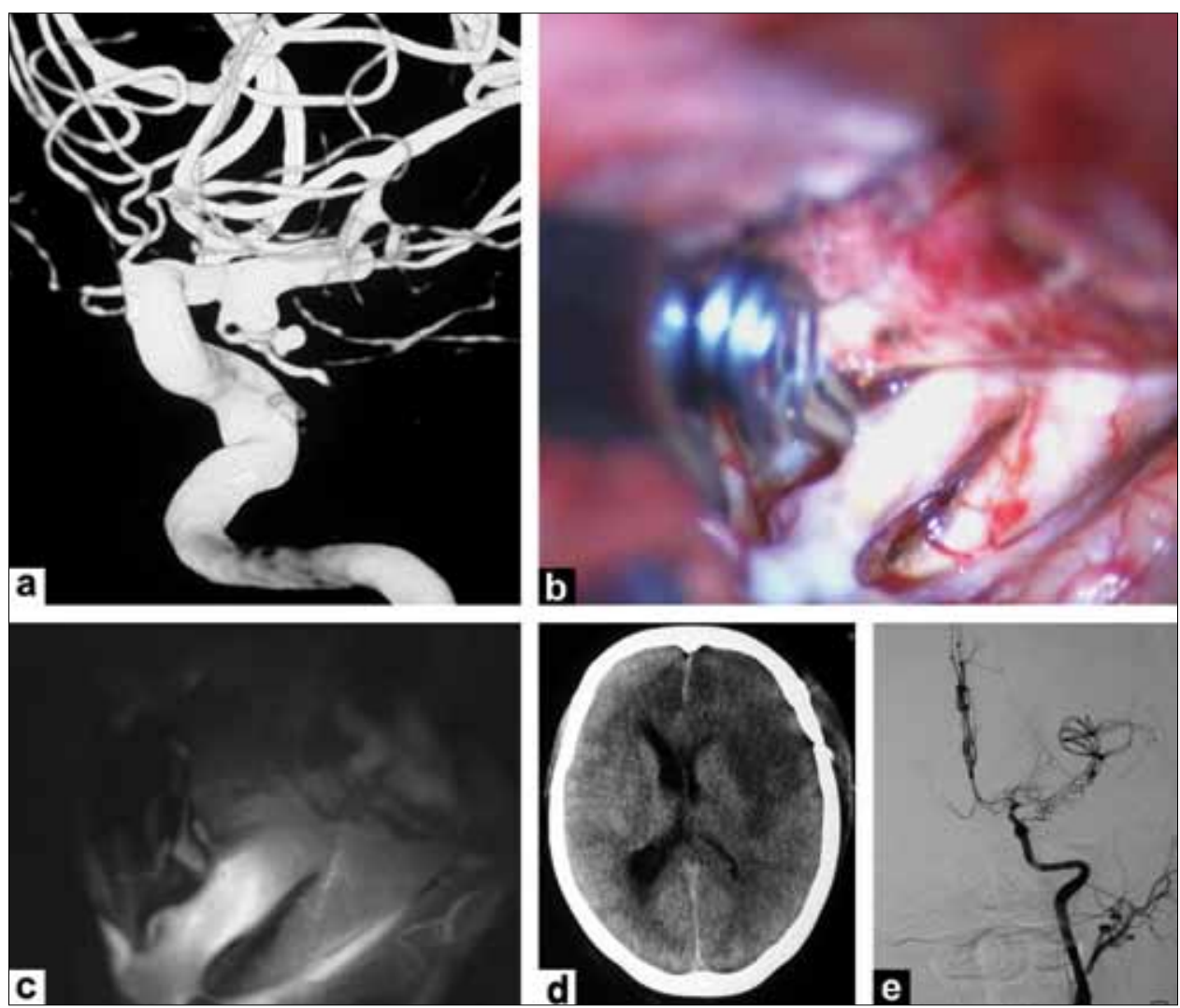

Figure 2: (a) Preoperative 3-D digital subtraction angiography showing the left posterior communicating artery aneurysm; (b) Intraoperative exposure of the aneurysm and clipping; (c) Indocyanine green angiography demonstrating complete aneurysmal clipping and the patency of the internal carotid artery and the anterior choroidal artery; (d) Postoperative CT showing the infarction of the frontal and temporal lobes; (e) Postoperative digital subtraction angiography showing severe vasospasm of left internal carotid artery

for the surgical procedure were compatible with those findings. However, as no intraoperative method is absolutely reliable, it makes sense to use a combination of methods, such as intraoperative DSA, Doppler ultrasonography, and electrophysiological monitoring to evaluate difficult cases.

\section{Conclusion}

Indocyanine green angiography with high time and spatial resolution is a simple, reliable and costeffective method. It provides real-time information in detecting the patency of parent, branching and perforating arteries. It can help to improve the quality and outcomes of surgical procedures. The method can be used routinely or as an adjunct to other methods. In patients with large or giant, wideneck aneurysms or with thick-walled atherosclerotic vessels, the ICG angiography may be unreliable. In such cases, ICG angiography combined with intraoperative DSA, microvascular Doppler ultrasound and electrophysiological monitoring may further improve the outcomes of intracranial aneurysm surgery.

\section{References}

1. Alexander TD, Macdonald RL, Weir B, Kowalezuk, Andrew BA. Intraoperative angiography in cerebral aneurysm surgerym A prospective study of 100 craniotomies. Neurosurgery 1996;39:10-8.

2. Chiang VL, Gailloud P, Murphy KJ, Rigamonti D, Tamargo RJ. Routine intra-operative angiography during aneurysm surgery. J Neurosurg 2002;96:988-92.

3. Raabe A, Nakaji P, Beck J, Kim LJ, Hsu FP, Kamerman JD, et al. Prospective evaluation of surgical microscope-integrated intraoperative near-infra-red indocyanine green videoangiography during aneurysm surgery. J Neurosurg 2005;103:982-9.

4. Raabe A, Beck J, Gerlach R, Zimmermann M, Seifert V. Nearinfrared indocyanine green video angiography: A new method for intraop-erative assessment of vascular flow. Neurosurgery 2003;52:132-9.

5. Raabe A, Beck J, Seifert V. Technique and image quality of intraoperative indocyanine green angiography during aneurysm surgery using surgical microseope integrated near-infrared video technology. Zentralbl Neurochir 2005;66:1-6.

6. MacDonald RL, Wallace MC, Kestle JR. Role of angiography following aneurysm surgery. J Neurosurgery 1993;79:826-32.

7. Kubo Y, Ogasawara K, Tomitsuka N, Otawara Y, Kakino S, Ogawa A. Revascular-ization and parent artery occlusion for giant internal carot-id artery aneurysms in the intracavernous portion using intraoperative monitoring of cerebral hemodynamics. Neurosurgery $2006 ; 58: 43-50$

8. Imizu S, Kato Y, Sangli A, Oguri D, Sano H. Assessment of incomplete clipping of aneurysms intraoperatively by a near-infrared indocyanine green-video angiography (Niicg-Va) integrated microscope. Mini Inva Neurosurg 2008;51:199-203. 
9. De Oliveira JG, Beck J, Seifert V, Teixeira MJ, Raabe A. Assessment of flow in perforating arteries during intracranial aneurysm surgery using intraoperative near-infrared indocyanine green videoangiography. Neurosurgery 2007;61:63-72.

10. Martin NA, Bentson J, Vinuela F, Hieshima G, Reicher M, Black K, et al. Intraoperative digital subtraction angiography and the surgical treatment of intracranial aneurysms and vascular malformations. J Neurosurg 1990;73:526-33.

11. Regillo CD. The present role of indocyanine green angiog-raphy in ophthalmolog. Curr Opin Ophthalmol 1999;10:189-96.

12. Woitzik J, Horn P, Vajkoczy,Schmiedek P. Intraoperative control of extracranial-intracranial bypass patency by near-infrared indocyanine green videoangiography. J Neurosurg 2005;102:692-8.

13. Payner TD, Horner TG, Leipzig TJ, Scott JA, Gilmor RL, DeNardo AJ. Role of intraoperative angiography in the surgical treatment of cerebral aneurysms. J Neurosurg 1998;88:441-8.

14. Dashti R, Laakso A, Niemelä M, Porras M, Hernesniemi J. Microscope-integrated near-infrared indocyanine green videoangiography during surgery of intracranial aneurysms: The Helsinki experience. Surg Neurol 2009;71:543-50.

Accepted on 02-07-2009

Source of Support: Nil, Conflict of Interest: None declared. 